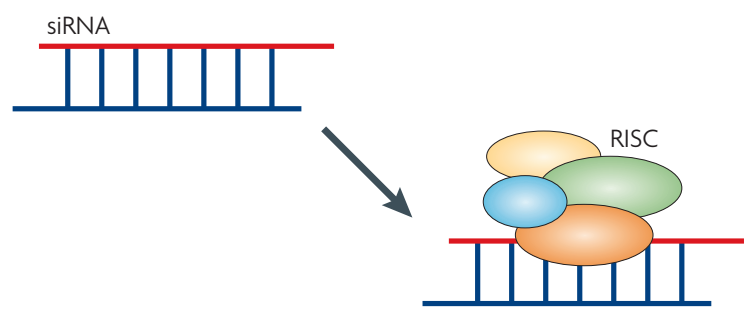

SMALL RNAS

\title{
Endo-siRNAs truly endogenous
}

Among animals, endogenous-small-interfering (endo-si) RNA pathways have so far been restricted to Caenorhabditis elegans. Seven studies now report the discovery of endo-siRNA pathways in Drosophila melanogaster and mouse oocytes.

During RNA interference (RNAi), members of the Dicer family of proteins cleave doublestranded (ds) RNA of an endogenous or exogenous source into 21-24 nucleotide siRNAs. dsRNA cleavage is coupled to the loading of these siRNAs onto the RNA-induced silencing complex (RISC). The siRNA guides the RISC complex to the corresponding target mRNA, which is degraded through cleavage by Argonaute (AGO) proteins.

The groups of Greg Hannon and Hiroyuki Sasaki found a large number of both Piwiinteracting RNAs (piRNAs) and endo-siRNAs that corresponded to mRNAs or retrotransposons in growing oocytes. Endo-siRNAs were exclusively mapped to retrotransposons or other genomic regions that produce transcripts capable of forming dsRNA structures. The genomic sources of dsRNAs that generate endo-siRNAs included inverted repeat structures, sites of bidirectional transcription and antisense transcripts from various loci, including retrotransposons and pseudogenes. Loss of Dicer or AGO2 resulted in decreased levels of endo-siRNAs and increased levels of retrotransposon and protein-coding transcripts that are complementary to the endo-siRNAs. These findings reveal a role for endo-siRNAs in mammals and indicate that the RNAi pathway regulates both protein-coding transcripts and retrotransposons in mouse oocytes.

Endo-siRNAs were also reported in D. melanogaster gonadal and somatic cells by the groups of Greg Hannon, Eric Lai, Haruhiko and
Mikiko Siomi, and Phil Zamore. Many endo-siRNAs correspond to transposons, heterochromatic sequences, and cis-natural antisense transcripts, and can target both protein-coding genes and mobile elements. Endo-siRNAs also derived from long hairpin RNA genes and target selected mRNAs in trans. Intriguingly, fly endo-siRNA production requires not only canonical RNAi factors (Dicer-2 and AGO2), but also the canonical microRNA biogenesis factor Loquacious. By contrast, piRNAs are thought to be generated from single-stranded precursor RNAs and their production in flies and vertebrates does not require Dicer. Loss of the endo-siRNAs correlates with loss of transposon silencing, which indicates that endo-siRNAs silence transposons in the fly soma, much as piRNAs silence transposons in the germ line.

Taken together, these studies suggest an evolutionary conservation of dsRNAs as regulatory molecules, a property previously ascribed only to microRNAs, and raise new regulatory possibilities for endogenous small RNA pathways.

Ekat Kritikou

ORIGINAL RESEARCH PAPERS Chung, W.-J. et al. Endogenous RNA interference provides a somatic defense against Drosophila transposons. Curr. Biol. 22 May 2008 (doi:10.1016/j.

cub.2008.05.006) |Czech, B. et al. An endogenous small interfering RNA pathway in Drosophila. Nature 7 May 2008 (doi:10.1038/ nature07007) | Ghildiyal, M. et al. Endogenous siRNAs derived from transposons and mRNAs in Drosophila somatic cells. Sciencexpress 10 April 2008 (doi:10.1126/science.1157396) | Kawamura, Y. et al. Drosophila endogenous small RNAs bind to Argonaute 2 in somatic cells. Nature 7 May 2008 (doi:10.1038/nature06938) |Okamura, K. et al. The Drosophila hairpin RNA pathway generates endogenous short interfering RNAs. Nature 7 May 2008 (doi:10.1038/ nature07015)|Tam, O. H. et al. Pseudogene-derived small interfering RNAs regulate gene expression in mouse oocytes. Nature 10 April 2008 (doi:10.1038/nature06904) |Watanabe, T. et al. Endogenous siRNAs from naturally formed dsRNAs regulate transcripts in mouse oocytes. Nature 10 April 2008 (doi:10.1038/ nature06908) 